\title{
Vitamin-C: properties, function and application in cancer therapy
}

\begin{abstract}
Vitamin-C or ascorbic acid is an excellent chain breaking type of antioxidant occupied in many biological processes in the body. It is a cofactor for the different type of metabolic enzymes. Vitamin-C has different properties such as anti-aging, anti scurvy, antiviral and antibacterial, enhancing immunity, involved in detoxification process. It has the ability to inhibit cancer cell proliferation by enhancing the immune system, stimulation of collagen synthesis, inhibiting the hyaluronidase and generate reactive oxygen or increased levels of $\mathrm{H}_{2} \mathrm{O}_{2}$, induction of apoptosis which damaged the Tümör cells but and it does not give the cytotoxic effect to the normal cell. Its anticancer effect is dose-dependent. In this review, highlight the properties, applications and beneficial effects of as corbate in cancer therapy and its prevention and its potential antitumor effects.
\end{abstract}

Keywords: ascorbic acid, anticancer, immunity enhancer, collagen
Volume 9 Issue 6 - 2018

Satish Shilpi,' Roshni Shivvedi, 'Amrita Singh,' Anil Kumar,' Gaurav K Saraogi, ${ }^{2}$ Vikas Jain, ${ }^{3}$ Kapil Khatri'

'Department of Pharmaceutics, Ravishankar College of Pharmacy, India

${ }^{2} \mathrm{NMIMS}$, School of Pharmacy and Technology Management, India ${ }_{3}^{3}$ SS College of Pharmacy, India Correspondence: Dr. Satish Shilpi,Associate Professor, Drug
Targeting Research Project Laboratory, Ravishankar College of
Pharmacy, Bypass Road, Bhanpur Square, Bhopal (MP), India, Tel
+91-9406520691, Email shilpesatish@gmail.com

Received: July 30, 2018 | Published: December II, 2018

\section{Introduction}

Vitamin-C also known as ascorbic acid and it is a water-soluble antioxidant and enzyme cofactor present in plants and some animals. Unlike most mammals, humans do not have the ability to synthesize this nutrient endogenously and, therefore, obtain it through diet. Ascorbic acid (AA) is a primary antioxidant remains in human plasma. ${ }^{1}$ While

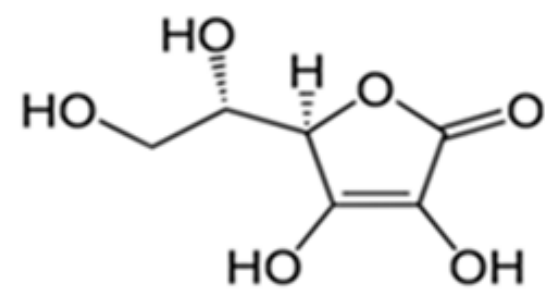

Ascorbic Acid

(AA) numerous animal and cell studies have shown beneficial anti-oxidant effects of AA. It is a chain breaking antioxidant and it give their effect by reacting with oxygen, hydroxyl, and superoxide radicals of the substances. It react with radicals of tocopheroxy to re-generate vitamin E. ${ }^{2}$ There are 2 chemical forms of Vitamin-C: the reduced form (ascorbic acid; AA) and the oxidized form (dehydroascorbic acid; DHA) (Figure 1). ${ }^{1,2}$

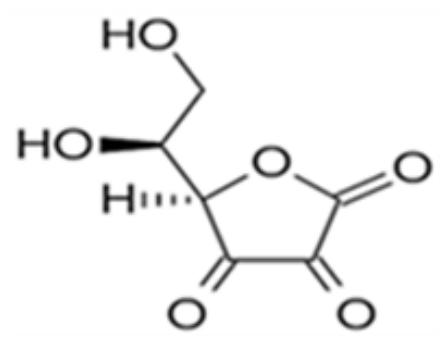

\section{De-Hydroascorbic Acid}

(DHA)

Figure I Structure of Ascorbic acid and De-Hydroascorbic acid.

The reduced form is approaches as an essential micronutrient involved in many biochemical and biological functions and is more predominant chemical structure in the human body. The maintenance of necessary concentrations of Vitamin-C to normal cellular metabolism involves 2 families of Vitamin-C transporters: glucose transporters (GLUTs) and sodium-coupled transporters (SVCTs). The transport of DHA is done through the GLUTs, mainly GLUT 1, 3, and 4, while the intake of AA is achieved through SVCT 1 and 2 (Table 1). Most of the tumour cells cannot transport AA directly to his interior, which is why these cells obtain Vitamin-C in its oxidized form. ${ }^{3,4}$

Table I Forms and types of transport of Vitamin-C

\begin{tabular}{lll}
\hline Forms & Vitamin-C & Principal transporters \\
\hline Reduced form & Ascorbic acid (AA) & Sodium-coupled transporters I and 2 (SVCTI and 2) \\
Oxidized form & Dehydroascorbic acid (DHA) & Glucose transporters I, 3 and 4 (GLUTI, 3 and 4) \\
\hline
\end{tabular}

Vitamin-C is a superior reducing agent, which form the ascorbate radical after two successive oxidations. Ascorbate is generally non reactive due to the presence of stable unpaired electron which oxidizes ascorbic acid to De-hydroascorbic acid (DHA). This reducing agent function is what maintains the structure of enzymes, thus allowing the biochemical machinery of cells and tissues functioning normally. ${ }^{4}$ 
Vitamin-C biosynthesised in different plant species (scheme-1) and it is a necessary nutrient which works as an antioxidant and it implicated in the tissue repair and production of enzymes of definite neurotransmitters. The functioning of several enzymes depends upon Vitamin-C. It play very important role to maintain the immune system of the body. ${ }^{1,3,4}$

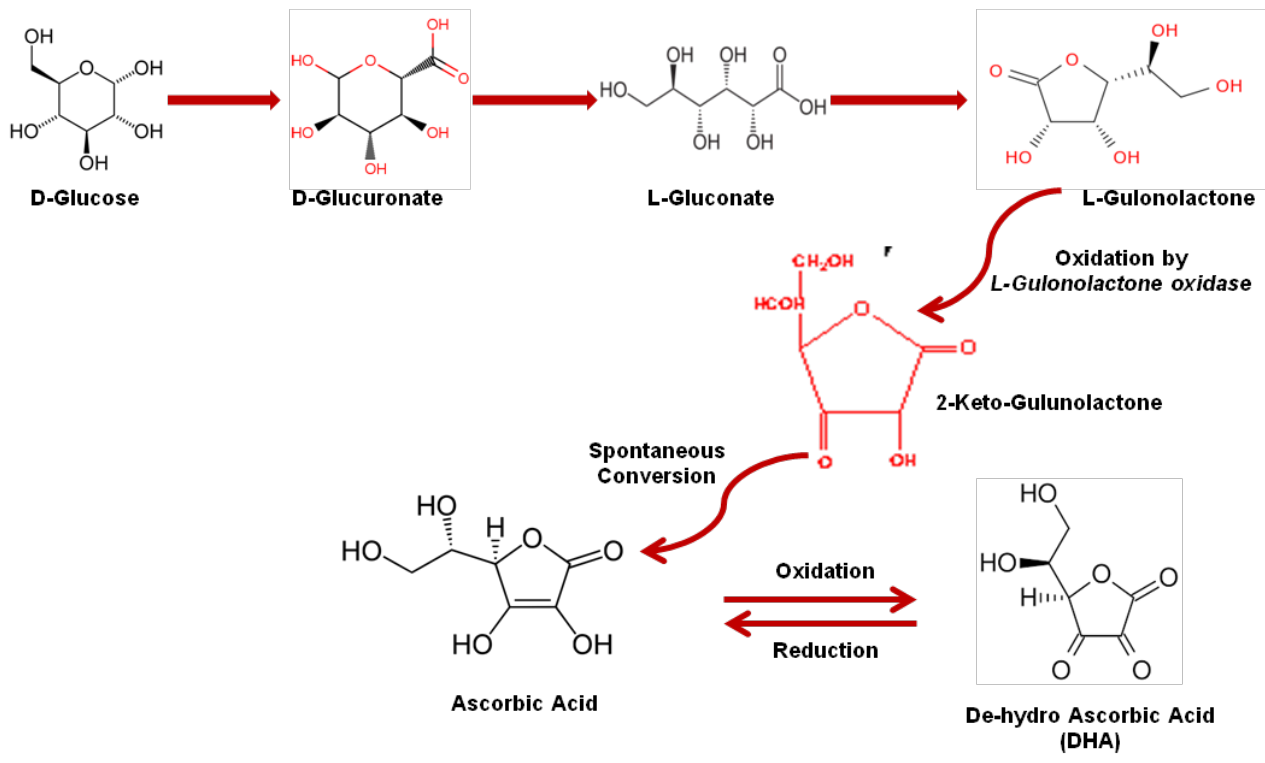

Scheme-I Biosynthesis and conversion of Vitamin-C to DHA.

\section{Function and working mechanism of vitamin-C}

The biological functions of ascorbic acid stem from its ability to provide reducing equivalents for a variety of biochemical reactions. Based on its reducing power, this Vitamin-Can reduce physiologically relevant reactive oxygen species. ${ }^{5}$ As a result, this vitamin primarily functions as a cofactor for reactions that require a reduced iron or copper metalloenzyme. It can also act as a protective antioxidant that operates in the aqueous phase both intra- and extra-cellular. ${ }^{6,7}$ Vitamin-C can minimize the cardio toxicity of adriamycin and can synergist the anticancer activity of some drugs such as cisplatin, dacarbazine, tamoxifen, doxorubicin and paclitaxel. In combination with anticancer drugs, it improves the immunity, tissue repair, detoxification process. Vitamin-C also has antiviral and antibacterial, by enhancing lympho-proliferation. It was revealed in previous studies Vitamin-C enhance the levels of interferon, antibody, hormones, ground substance. ${ }^{3,5,7}$

Vitamin-C increase the production of lymphocytes in the body resulting in the enhancing the immune system. ${ }^{8}$ It has observed in the cancer patient, the lymphocytes ascorbate level is found low. Lymphocytes are necessary to prevent initiating phase of the cancer cell growth. Aadequate ascorbate level in the body enhances the production and effectiveness of lymphocytes and also enhances the phogocytosis of the oncogenic viruses or other oncogenic cells. van Gorkom et al., ${ }^{8}$ studied in vitro to the effect of Vitamin-C in enhancing the lymphocyte production in the body. The study found that ascorbic acid require to the development of $\mathrm{T}$ cell, its proliferation and function. It was found that it enhanced the propagation of a group of cytotoxic natural lymphocytes (NK cells). Vitamin-C increase the immune reconstitution of cancer patients treated with immunotoxic drugs. ${ }^{8}$

It plays a major role in the formation of collagen which stimulation of collagen formation which walling-off or develop impermeable barrier (Schirrus) to the tumors in which. It plays an important role in collagen synthesis. It increases the hydroxylation of proline and lysine to hydroxyproline and hydroxylysine, respectively, and proper crosslinking of these amino acid is occur which result in stable triple helix form of collagen. The triple helix collagens provide stability to for stable barrier surrounding the tumor mass that prevent the metastasis of tumor. ${ }^{5,7,8}$

It can also inhibit the hyaluronidase and develop the environment of the ground substance to make the tumor intact and preventing metastasis. It reduces the toxicity associated with chemotherapeutic agents by reducing the cellular free radical damage and also it reduces the chances of cancer by neutralizing the carcinogenic substances. Due to the effectiveness of Doxorubicin as anti-cancer agents, its regular use may cause dose-dependent cardiotoxicity which is a serious life-threatening apprehension. ${ }^{3}$ Akolkar et al., ${ }^{9}$ studied the effect of Vitamin-C to mitigate the doxorubicin-induced cardiomyopathy. They were isolated Cardiomyocytes from adult Sprague-Dawley rats which were pre-treated with Vitamin-C $(25 \mu \mathrm{M})$, Doxorubicin $(10 \mu \mathrm{M})$ and Vitamin-C $(25 \mu \mathrm{M})+$ Doxorubicin $(10 \mu \mathrm{M})$ for 24 hours Vitamin-C alleviate and checked levels of nitric oxide in cardiomyocyte. They found that Vitamin-C pre-treatment reduced the level of cellular nitric oxide due to downregulation of inducible nitric oxide synthase (iNOS) expression and endothelial nitic oxide synthase (NOS) activity resultant the decreasing peroxynitrite formation in doxorubicintreated cardiomyocytes. They conclude that Vitamin-C can be used to mitigate the cardiotoxic side effects caused by doxorubicin. ${ }^{9}$

\section{Vtamin $\mathbf{C}$ as an anticancer agent}

The activity of Vitamin-C depends mainly on its concentration in plasma. Chen et al., ${ }^{10}$ studied and revealed that when the concentration of ascorbate in plasma is reached more than $1 \mathrm{mM}$, at this concentration, it behaves like pro-oxidant. It occurs in the presence of transition metal like copper and iron which reduced by ascorbate and then reacts with hydrogen peroxide $\left(\mathrm{H}_{2} \mathrm{O}_{2}\right)$. It leads to generate very highly reactive hydroxyl radicals which are capable to damage or kill the tumor cell (Figure 2). ${ }^{10-12}$ 


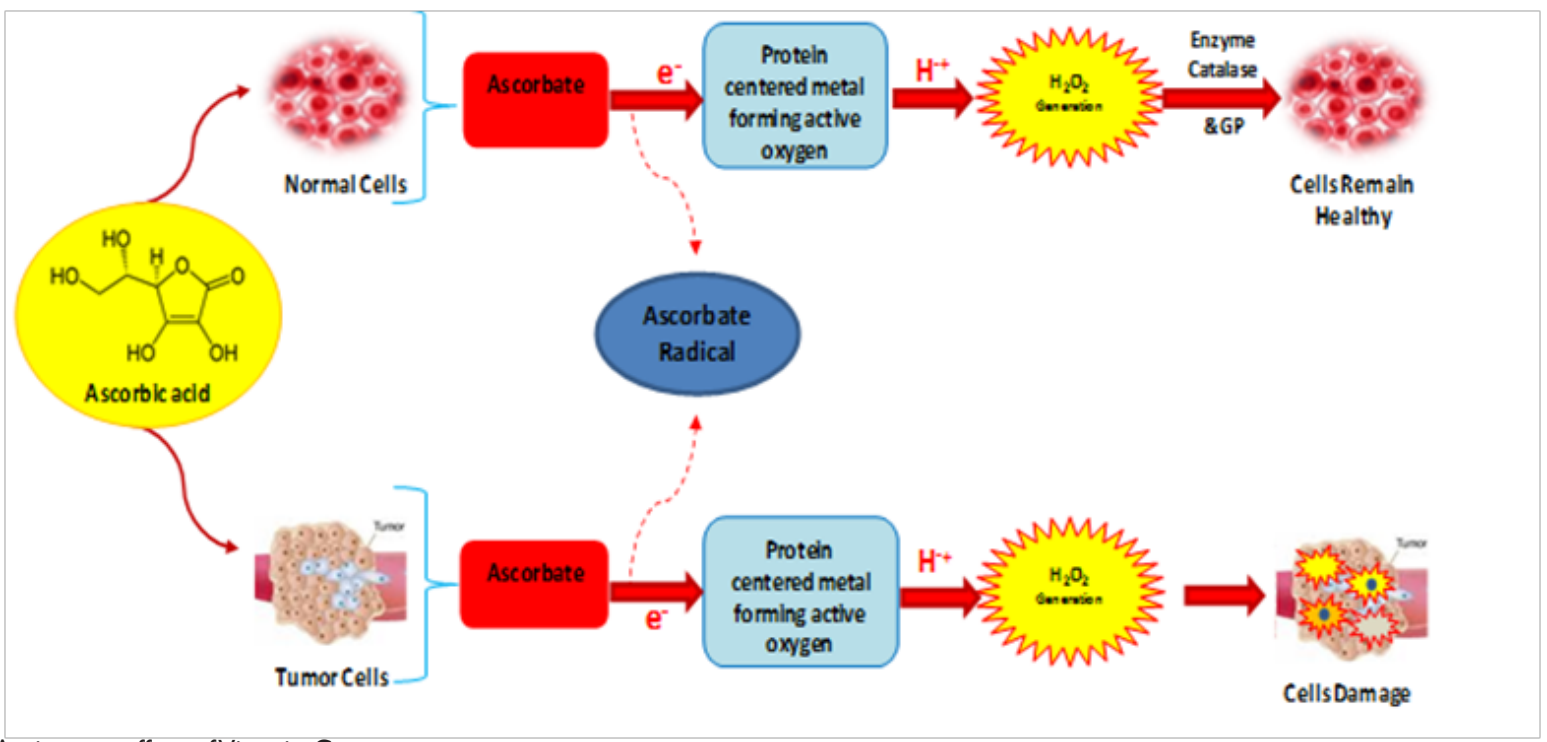

Figure 2 Anticancer effect of Vitamin-C.

But in case of normal cells, $\mathrm{H}_{2} \mathrm{O}_{2}$ destroyed immediately due to the presence of catalase and Glutathione peroxidase (GP) (both are a class of antioxidant enzymes) in high concentration due to high blood flow. While they have sufficient it induces hydrogen peroxide $\left(\mathrm{H}_{2} \mathrm{O}_{2}\right)$, which is preferentially toxic toward tumour cells. In the case of tumour cells, the concentration of antioxidant enzymes is very low due to low blood perfusion and the $\mathrm{H}_{2} \mathrm{O}_{2}$ remains active in the cells that damage the cells. ${ }^{3,6,10,12,13}$

It is a very important fact that the antitumor effect of ascorbate due to apoptosis, pyknosis and necrosis process are mainly dependent on extracellular ascorbate concentration but these processes not occur with intracellular ascorbate. Antitumor effect of ascorbate may include high rate of apoptotic pathways, and elevated pro-oxidant damage of the tumor cells that cannot be restorable as well as oxidation of ascorbate in plasma at high level form unstable metabolite i.e. dehydroascorbic acid, which is very toxic to tumour cells. ${ }^{14,15}$

\section{Application of Vitamin-C in cancer therapy}

Use of Vitamin-C for cancer therapy was popularized by Linus Pauling. At high concentrations, ascorbate is preferentially toxic to cancer cells. There is some evidence that large doses of Vitamin-C, either in multiple divided oral doses or intravenously, have beneficial effects in cancer therapy. ${ }^{16,17}$ Oral doses, even in multiple divided doses, are not as effective as intravenous administration. Vitamin-C at a dose of $1.25 \mathrm{~g}$ administered orally produced mean peak plasma concentrations of $135 \pm 21 \mu \mathrm{mol} / \mathrm{L}$ compared with $885 \pm 20 \mu \mathrm{mol} / \mathrm{L}$ for intravenous administration (Padayatty et al., 2004). Most leukemia patients with TET2 mutations or deletions exhibit the mono-allelic TET2 loss, and disease progression in Tet $2+/$ and Tet $2 /$ mice is almost indistinguishable indicating that even a $50 \%$ loss of TET2 function can initiate aberrant hematopoiesis. ${ }^{18}$ An et al., ${ }^{19}$ have found that Vitamin-C impairs aberrant re-plating capacity and myeloid disease progression in hematopoietic stem and progenitor cells (HSPCs) even in the complete absence of functional Tet2 ${ }^{19}$ Recent studies have shown that Tet 2 and Tet 3 exhibit overlapping tumour suppressive roles, as both are highly expressed in myeloid progenitors and their combined deletion leads to rapid and aggressive AML. ${ }^{19}$ TET3 activation might compensate sufficiently for lack of TET2 to diminish aberrant self-renewal upon Vitamin-C treatment. Indeed, we find that combined Tet $2 /$ Tet 3 deficiency causes severe depletion of steady- state 5-hydroxymethylcytosine $(5 \mathrm{hmC})$ in HSPC DNA, diminished capacity for $5 \mathrm{hmC}$ generation in response to Vitamin-C, and markedly decreased efficacy of Vitamin-C in suppressing aberrant selfrenewal. ${ }^{16}$ These findings suggest that Vitamin-C acts predominantly through TETs, and accordingly, a minimal amount of TET activity is required for Vitamin-C action. ${ }^{20-23}$ Yun et al., ${ }^{21}$ presented data stating that oxidized Vitamin-C was able to kill colorectal cancer cells (CRC) depending on the KRAS mutational status. ${ }^{21}$ They found that cultured human CRC cells harboring KRAS or BRAF mutations were selectively killed when exposed to high levels of vitamin- C. This effect was due to increased uptake of the oxidized form of Vitamin-C, dehydroascorbate (DHA), via the GLUT1 glucose transporter. Increased DHA uptake caused oxidative stress when intracellular DHA is reduced to Vitamin-C, depleting glutathione. Thus, ROS accumulate and inactivate glyceraldehyde 3-phosphate dehydrogenase (GAPDH), an enzyme of $\sim 37 \mathrm{kDa}$ that catalyzes the sixth step of glycolysis and thus serves to break down glucose for energy and carbon molecules. Inhibition of GAPDH in highly glycolytic KRAS or BRAF mutant cells leads to an energetic catastrophe and cell death not seen in normal cells. ${ }^{21}$ In this work, author described a novel antitumoral mechanism of Vitamin-C in KRAS mutant colorectal cancer involving the metabolic disruption through downregulation of key metabolic checkpoints in KRAS mutant cancer cells and tumors without killing human immortalized colonocytes. ${ }^{22}$ Vitamin-C is capable to induce RAS detachment from the cell membrane via ROS inhibition. Thus, RAS detachment leads inhibition ERK 1/2 and PKM2 phosphorylation. As a consequence of this activity, we could observe strong downregulation of the glucose transporter (GLUT1) and pyruvate kinase M2 (PKM2)-PTB dependent protein expression causing a major blockage of the Warburg effect and therefore energetic stress. Tumor-specific pyruvate kinase M2 (PKM2) is a master regulator for the Warburg effect and In addition to its well-established role in aerobic glycolysis, PKM2 directly regulates gene transcription. ${ }^{23}$ Vitamin-C extends to human lung adenocarcinoma SPC-A-1 cells. A combination of the flavonoid, epigallocatechin gallate, and Vitamin-C increased the number of cells in the G0/G1 cell cycle. The inhibition of proliferation of SPC-A-1 cells was determined to be synergistic. ${ }^{11}$ Guerriero et al., ${ }^{15}$ investigated the response when VitC is added to the breast cancer drug, mitoxantrone (MTZ) on MDA-MB231 and MCF7 breast tumor cell lines. The MDA-MB231 is a highly metastatic cell line which exhibits alpha-negative estrogen receptors, and fibroblast- 
like properties. ${ }^{12}$ MCF7 has estrogen receptor alpha, is less metastatic, and is characterized as luminal epithelial like. After treating cells with different doses of Vitamin-C as well as MTZ, it was shown that the combination of Vitamin-C and MTZ significantly decreased the cell viability of the breast cancer cell lines. found a synergistic effect on the median inhibitory concentration (IC50) with it being lowered in Vitamin-C/MTZ combination trials. Moreover, the data showed a slight increase in the G2/M elongation and the activation of H2AX and PI3K pathways. This is important because the H2AX pathway is involved in DNA repair at checkpoints and through a chain of signaling may arrest cell progression. Additionally, $\mathrm{H} 2 \mathrm{AX}$ has been associated with several double stranded DNA breaks. ${ }^{12}$ The use of Vitamin-C can kill cancer cells at pharmacologic levels, the aforementioned studies show that it has an extended use as a 'helping' molecule. ${ }^{10}$

\section{Conclusion}

Ascorbic acid is naturally occurring a substance has different properties including anticancer, antioxidant, anti-aging and immunity enhancing etc. It is not cytotoxic towards normal cells but it shows cytotoxicity to cancer cells. It may extend the survival and improve the quality of life of patient suffered from cancer and if it is given with the other anticancer agents than it can synergist the anticancer action of some other potent anticancer drugs.

\section{Acknowledgment}

Author want to acknowledge the Science and Engineering Research Board (SERB), Department of Science and technology(DST), New Delhi, India and Madhya Pradesh Council of science and Technology (MPCST) for awarding research project and providing financial assistance to carry out the research work on advanced breast cancer and liver cancer.

\section{Conflicts of interest}

Author declares that there is no conflicts of interest.

\section{References}

1. Frei B, England L, Ames BN. Ascorbate is an outstanding antioxidant in human blood plasma. Proc Natl Acad Sci USA. 1989;86:6377-6381.

2. Heller R, Munscher-Paulig F, Grabner R, et al. L-Ascorbic acid potentiates nitric oxide synthesis in endothelial cells. J Biol Chem. 1999;274(12):8254-8260.

3. Heaney ML, Gardner JR, Karasavvas N, et al. Vitamin C antagonizes the cytotoxic effects of antineoplastic drugs. Cancer Res. 2008;68:8031-8038.

4. Rivas CI, Zuniga FA, Salas-Burgos A, et al. Vitamin C transporters. $J$ Physiol Biochem. 2008;64(4):357-375.

5. Buettner GR, Jurkiewicz BA. Ascorbate free radical as a marker of oxidative stress: An EPR study. Free Radic Biol Med. 1993;14:49-55.

6. Englard S, Seifter S. The biochemical functions of ascorbic acid. Annual Review of Nutrition. 1986;6:365-406.
7. Whiteman M, Jenner A, Halliwell B. Hypochlorous acid-induced base modifications in isolated calf thymus DNA. Chem Res Toxicol. 1997;10:1240-1246.

8. Van Gorkom Gny, Klein Wolterink RGJ, Van Elssen Chmj, et al. Influence Of Vitamin C On Lymphocytes: An Overview. Antioxidants (Basel). 2018;7(3):E41

9. Akolkar G, Bagchi A, Jassal D, et al. Vitamin C reduces doxorubicininduced nitrosative stress by regulation of nitric oxide synthase. Canadian Journal of Cardiology. 2015;31(10):S66.

10. Chen Q, Espey MG, Krishna MC, et al. Pharmacologic ascorbic acid concentrations selectively kill cancer cells: action as a prodrug to deliver hydrogen peroxide to tissues. Proc Natl Acad Sci US. 2005;102:13604-13609.

11. Kathleen A, Head ND. Ascorbic Acid in the Prevention and Treatment of Cancer. Altern Med Rev. 1998;3(3):174-186.

12. Ana Maria Oliveira Ferreira da Mata, Ricardo Melo de Carvalho, Marcus Vinícius Oliveira Barros de Alencar, et al. Ascorbic acid in the prevention and treatment of cancer. Rev Assoc Med Bras. 2016;62(7):680-686.

13. Lee WJ. The Prospects of Vitamin $\mathrm{C}$ in Cancer Therapy. Immune Netw. 2009;9(5):147-152.

14. Li W, Wu J, Tu Y. Synergistic effects of tea polyphenols and ascorbic acid on human lung adenocarcinoma SPC-A-1 cells. J Zhejiang Univ Sci B. 2010;11(6):458-464.

15. Guerriero E, Sorice A, Capone F et al. Vitamin-C effect on mitoxantroneinduced cytotoxicity in human breast cancer cell lines. PLoS One. 2014;9:e115287.

16. Ohno S, Ohno Y, Suzuki N, et al. High-dose Vitamin C (Ascorbic Acid) Therapy in the Treatment of Patients with Advanced Cancer. Anticancer Res. 2009;29(3):809-815.

17. Riordan $\mathrm{HD}$, Hunninghake $\mathrm{RB}$, Riordan $\mathrm{NH}$, et al. Intravenous ascorbic acid: protocol for its application and use. $P R$ Health Sci J. 2003;22(3):287-290.

18. Delhommeau F, Dupont S, Della VV, et al. Mutation in TET2 in myeloid cancers. N Engl J Med. 2009;360(11):2289-2301.

19. An J, Gonza lez-Avalos E, Chawla A, et al. Acute loss of TET function results in aggressive myeloid cancer in mice. Nat Commun. 2015;6:1007.

20. Cimmino L, Dolgalev I, Wang Y, et al. Restoration of TET2 function blocks aberrant self-renewal and leukemia progression. Cell. 2017;170(6):1079-1095.e20

21. Yun J, Mullarky E, Lu C, et al. Vitamin-C selectively kills KRAS and BRAF mutant colorectal cancer cells by targeting GAPDH. Science. 2015;350(6266):1391-1396.

22. Aguilera O, Muñoz-Sagastibelza M, Torrejón B, et al. Vitamin-C uncouples the Warburg metabolic switch in KRAS mutant colon cancer. Oncotarget. 2016;7:47954-47965.

23. Yang W, Xia Y, Hawke D, et al. PKM2 phosphorylates histone $\mathrm{H} 3$ and promotes gene transcription and tumorigenesis. Cell. 2012;150(4):685-696. 